\title{
Management of squamos cell carcinoma of tongue in young men: case report
}

\author{
Israyani $^{1 *}$, Ayu F. Argadianti ${ }^{2}$, Hening Tuti Hendarti ${ }^{2}$, Adiastuti EP $^{2}$
}

${ }^{1}$ Department of Oral Medicine, Faculty of Dentistry, Hasanuddin University, Makassar, Indonesia ${ }^{2}$ Department of Oral Medicine, Faculty of Dental Medicine, Universitas Airlangga, Surabaya, Indonesia

${ }^{*}$ Correspondence to: israyani14@gmail.com

Received: 6 March 2019 Revised: 20 March 2019 Accepted: 25 March 2019 Available Online: 1 May 2019

\section{Abstract}

Objective: Oral squamous cell carcinoma is the most common malignant neoplasm of the oral cavity, usually affecting individuals over 50 years of age. It rarely occurs in patients who are less than 40 years old.

Methods: This case report aim is to focuses on establishment of diagnosis, differential diagnosis, predisposition, and treatment planning related to the case. This report describes a case of oral squamous cell carcinoma, staged T1N1M0 (stage III), involving the lateral border and ventral surface of the tongue of a 32-year old male patient, with no smoking or drinking habits. Initial tumor

\section{presentation was of deep ulceration and intense pain.}

Results: Patient has examination with autofluorence LED light, presence of bacterial growth and FNA for establised diagnosis. Patient was given chlorine dioxide mouthwash and education for further treatment one of them is tomoterapi.

Conclusion: Clinical features of deep ulceration, pain and induration may be a suspicious feature of oral cavity cancer, therefore early diagnosis of oral squamous cell carcinoma determines prognosis.

Keywords: Autofluoresence, Malignant, Tomoterapi

Cite this Article:Israyani, Argadianti FA, Hendrati TH, Adiastuti EP. 2019. The aesthetic management of gingival enlargement and hyperpigmentation in maxillary anterior region: a case report. Journal of Case Reports in Dental Medicine. 1(1): 21-26.

\section{Introduction}

Oral cavity cancer or oral carcinoma is a term often used to indicate malignancy in the oral cavity. The prevalence of oral carcinoma is high and be one of ten and even one of the six biggest causes of death. Cancer can be defined as uncontrolled tissue growth, which is the result of an imbalance between cell division and cell death that should occur. ${ }^{1-3}$ Oral Squamosa Cell Carcinoma (OSCC) defined as a malignant epithelial neoplasm which shows squamous differentiation characterized by keratin formation and / or the presence of an intercellular bridge. OSCC is the most common neoplasm in the oral cavity. OSCC usually occurs on the lateral side, ventral surface of the tongue and lips followed by the floor of the mouth, gingiva and alveolar mucosa and palate. ${ }^{3}$

OSCC is most common in men than women $(\mathrm{M}: \mathrm{F}=1.5: 1)$ possibly more men have high-risk habits than women. The development of the OSCC is in accordance with the period of exposure to risk factors, and in accordance with increasing age associated with changes in mutagenic dimensions (properties of materials that can change genetically altered chromosomes) and epigenetics (studies of phenotypic changes or genetic expression caused by mechanisms other than sequence changes base of DNA).

Data in the United States shows the average age of having OSCC around 62 years. However, the current OSCC incident is increasing at the age of under 45 years. $^{4}$

World Health Organization (WHO) data's in 2008 shows that 7,6 million people died of cancer, $70 \%$ of the cases occur in developing country and only $30 \%$ were successfully handled. Based on data of the Basic Health Research (Riskesda) in 2007 published by the Ministry of Health (2008) showed that the prevalence of head and neck cancer in Indonesia is quitely high, which ranks fourth in all cancer malignancies found in men and women.,

Etiologies and predisposition factors of oral cancer include tobacco use, alcohol, chewing of betel quid and areca nut, virus ( the most common cause is the human pappiloma virus), food (one of them is red chili powder and vitamin A deficiency), family history with Head and Neck Squamous Cell Carcinoma (HNSCC), molecularly it is reported that $50-70 \%$ of tumors in humans are due to p53 mutations, trauma, orodental factors, radiation. ${ }^{2,6,7}$

Oral cancer is usually diagnosed when it reaches its final stage, resulting in death rates in more than $50 \%$ of cases. OSCC cannot be diagnosed only clinically, but also requires enforcement through histology. ${ }^{8}$

In this case report, the diagnosis will be made to find out the existence of OSCC in young male patients under 40 years which begins with chronic trauma of the tongue. 


\section{Case Report}

\section{First visit, May 30rd 2017 ( Day-1)}

Male patient 32 years old, living in Surabaya came to Dental Hospital Airlangga University with complaints on the right side of the tongue there were white area and pain. The complaint was felt since four months ago. Complains began of the right side of the tongue feels uncomfortable because it kept rubbing against caries of the upper right posterior teeth. Then the tooth was removed at the dentist's practice about three months ago and two weeks later, the patient returned to the dentist's control and cleaned the teeth but there was no change. One month ago the patient complained about the right side of the tongue, white patches that were painful and felt hard when palpated. The pain spreads to the right throat and the patient found it difficult to move the tongue to swallow. One week ago the patient went to an internal medicine doctor and prescribed bacticin medicine and aloclair mouthwash but did not improve. Then the patient went to the dentist in one of the clinics in Surabaya and only cleaned the calculus but the spot had no changes and then the patient was advised to go to Dental Hospital Airlangga University. Patient claimed that he does not consume alcohol or smoke, and there is no family history known to have experienced the same thing.

On extra oral examination found desquamation on the lower lip, normal color, normal surrounding tissue, easily peeled off and not painful. On examination of the submandibularis gland there were nodules, solid consistency and pain.

On intra-oral examination found in the anterior and posterior gingiva of the mandible in the form of plaque, whitish color, normal surrounding tissue, can be scraped, and not painful. On the right ventrolateral tongue is found ulcer, solitary, $3 \times 4$ $\mathrm{mm}$ in size, irregular edges, clear borders, normal surrounding area, reddish surface, pain and induration. In addition, white plaques were also found, could not be scraped, no pain, clear boundaries of the same size as the ulcer are $3 \times 4 \mathrm{~mm}$. The dorsal tongue was found pseudomembrane, yellowish white in $2 / 3$ dorsal tongue, not painful. On the floor of the mouth was found nodular, clear boundaries, solid consistency, hard, slippery texture, the same color with bilateral surrounding tissue. Velscope was examined in patient.

The working diagnosis for the condition is chronic traumatic ulcer with a differential diagnosis of oral squamous cell carcinoma on the tongue, on on the lateral of the tongue. The patient was referred to the clinical pathology laboratory for complete blood examination, referred to for microbiological examination for bacteriological examination, suggested for examination of anatomical pathology, and planned for examination at the oncology clinic.

Occlusal grinding was performed on dental lingual cups $45,46,47$ of the patient. Velscope examination is also performed in patients with a reddish-orange picture that shows a bacterial or fungal infection (Figure 1.c). The patient is given a prescription for chlorine dioxide mouthwash of 160 $\mathrm{ml}$ of 1 bottle to be cured 4 times a day as much as 5 $\mathrm{ml}$ after meals. Patient is encouraged to start using the drug after the laboratory examination is done. In patients with IEC, namely to use drugs that are regularly prescribed, improve oral hygiene, limit the use of the right tongue by consuming highcalorie high-protein soft foods directed to the left oral cavity, rehydration, and control 1 week later.

\section{Second Visit, Control 1, June 6th 2017 ( day-8)}

On this second visit, the patient said that the right tongue felt better, was able to move the tongue a little even though it was still limited due to illness. Patient claimed to use drugs was prescribed regularly. The medicine has run out. Patient also came with the results of clinical pathology laboratory results with 80 segment results (Normal value: 54-62), low lymphocytes (Normal value: 25-33). On microbiological examination the culture showed results found positive for Streptococcus viridans (normal flora), and on microbiological examination gram staining was found to be gram positive bacteria. No pathogenic germs found.

On extra oral examination, examination of the submandibularis gland was nodules, solid consistency and still painful. On intra-oral examination of the right ventrolateral tongue, ulcer, solitary was found, measuring $3 \times 4 \mathrm{~mm}$, irregular edges, clear borders, normal surrounding area, reddish surface, pain and induration. In addition, white plaques were also found, could not be scraped, did not hurt, clear borders of the same size as the ulcer are $3 \times 4 \mathrm{~mm}$.

Working diagnosis for conditions above chronic traumatic ulcers with differential diagnosis of oral squamous cell carcinoma on the tongue, coated tongue, exostosis and creanated tongue. Patient was advised to examine anatomical pathology at the oncology clinic General Hospital Dr. Soetomo, but on the family's advice, the patient has made an appointment to conduct a self-examination to the doctor of anatomical pathology practice on June 9, 2017. In patients given a prescription for mouthwash chlorine dioxide mouthwash $160 \mathrm{ml}$ as much as 1 bottle to be delivered 4 times a day after $5 \mathrm{ml}$ eat. Patient was given IEC to use medication 

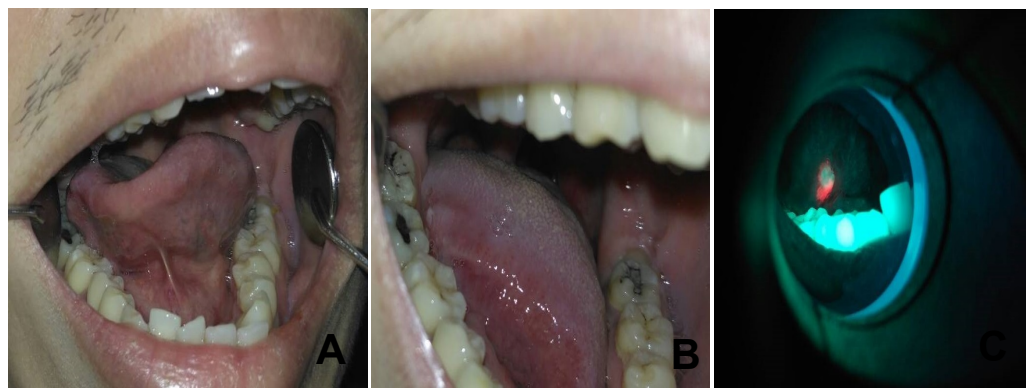

Figure 1 A and $B$. Ventrolateral ulcer of the tongue, single, size $3 \times 4 \mathrm{~mm}$, irregular edge, yellowish white surface, diffuse boundary, surrounding tissue color normal, sore and induration. $C$. The description of the velscope results shows a reddish orange color indicating a bacterial or microorganism infection.
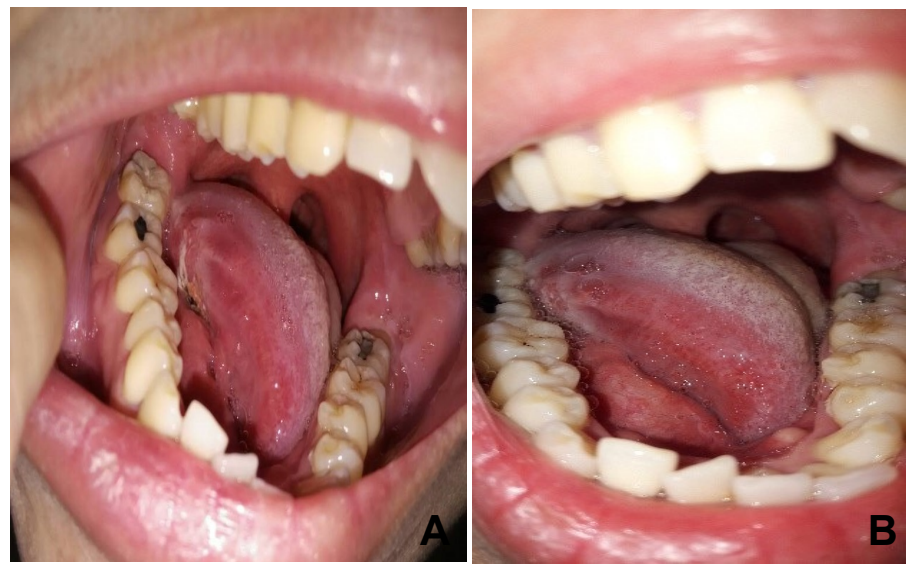

Figure 2 A and B. Ventrolateral ulcer of the tongue, single, size $3 \times 4 \mathrm{~mm}$, irregular edge, reddish white surface, diffuse boundary, surrounding tissue color normal, painful and induration.

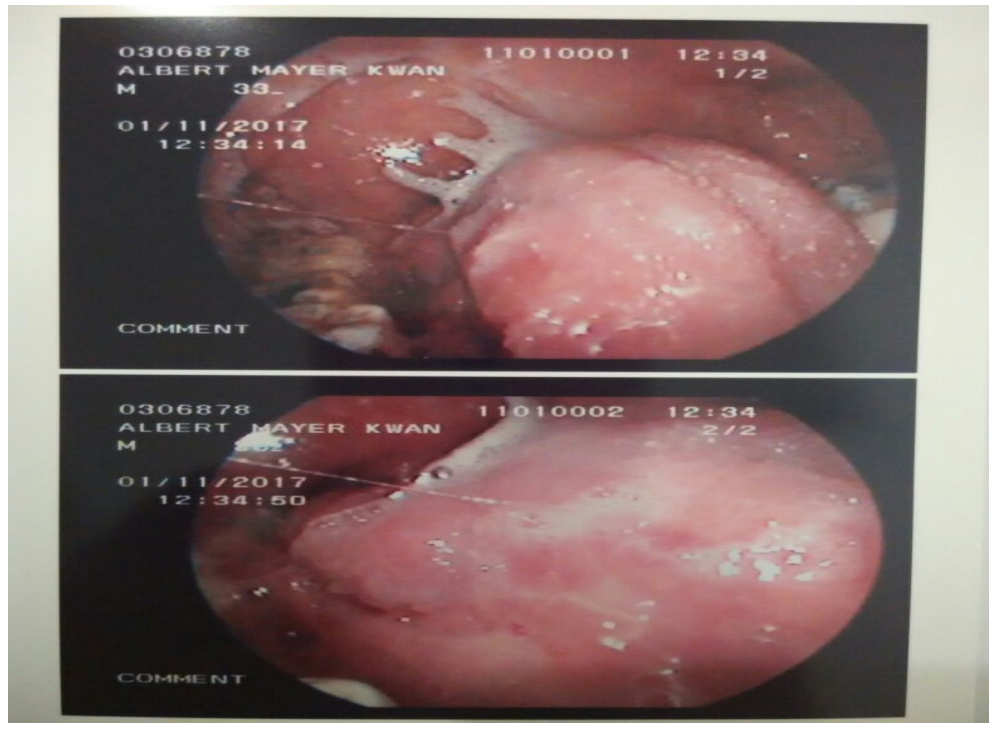

Figure 3 Right ventrolateral tongue after surgery regularly, improving oral hygiene, limiting the use of the right tongue, using orthodontic wax while sleeping on the right sleeping on the right posterior teeth cups, rehydration, adequate rest, eat high-calorie high-protein foods. Control one week later.

The patient did not come at the Dental Hospital Faculty of University Airlangga University but giving information through cellular media revealed the results of anatomical pathology examination which stated that patients who had aspiration cytology on FNA dextra colli tumor showed smear results showed fibrinous mass and red blood cell distribution, lymphoid cells and many cells anaplastic with a round to hyperchromatic oval nucleus, a little to broad bluish cytoplasm with DiffQuik, and a lot of keratin mass. Conclusion of these results that in dextra colli tumors, FNS showed metastatic Squamous cell carcinoma keratinizing type. The patient stated that he planned to continue treatment in Malaysia on family advice.

Information given by patient's wife that the patient continued treatment at one hospital in Penang, by undergoing tomoterapi 30 times and chemotherapy 5 times. The surgical results and information provided were post surgical patients on CT Neck with the result of heterogeneous increased in the tongue, advised to keep control again, reduced right cervical lymphadenopathy, thickening of the mucosa in the right maxillary sinus, streaky thickening of the right neck, possible postoperative changes.

The final diagnosis of this case is stage III oral squamous cell carcinoma on the ventrolateral tongue. Patient is advised to continue treatment and improve oral hygiene during treatment in the field of oncology.

\section{Discussion}

OSCC has been mentioned as a condition that rarely occurs in young adults. ${ }^{9}$ This case shows that patient are under the age of 45 years where the prevalence in OSCC patients at that age is still small, but in recent years it has been increasing at the age of under 45 years. The etiology mentioned as the cause of most oral cavity cancers in men is consumption of alcohol and smoking (tobacco), ${ }^{10}$ but the patient said that he did not use or consumer the two ingredients, including family history, no one experienced a similar condition. It is possible to be a cause, namely a genetic change or mutation, but no further examination is carried out on the patient, for example by the polymerase chain reaction method, which is a method used to produce a large number of specific DNA groups in a short period of time is a technique used to analyze changes in DNA mobility 
or differences in DNA electromobility. ${ }^{11}$

Cancer cells have characteristics that are different from normal cells and can proliferate uncontrollably, while normal cells proliferate in carrying out their functions to replace cells that have been damaged or that have died and are driven by growth factors produced by other cells. In cancer cells autocrine mechanisms occur, namely cells that produce their own growth factors so that proliferation is not controlled. Cancer cells experience rapid growth and the morphology is very different from normal cells. This explains that cancer cells are almost entirely composed of cell nuclei and only a few contain cytoplasm, besides that the morphological changes in cancer cells occur because of the pleiomorphic effect of transcription factors which in addition to affecting transcription of genes involved in cell replication also affect other genes. ${ }^{11}$

Carcinogenesis is a genetic process that can cause changes in molecular function, cell morphology and ultimately changes in cellular behavior. This process is not only confined to the epithelium but also involves complex epithelium, connective tissue, and interaction of immune functions. The main genes involved in OSCC include proto-oncogenes and tumor suppressor genes (TSGs). Genetic molecular regulation is also involved. Genetic changes can show loss or addition of alleles on chromosome regions that are compatible with proto-oncogenes and TSG, or epigenetic changes such as DNA methylation. ${ }^{1,12}$

OSCC appears as a result of various molecular events that cause genetic damage that affects chromosomes and genes, which eventually leads to DNA changes. The accumulation of these changes triggers cell dysregulation at the boundary where autonomic growth and invasive development occur. The neoplastic process initially manifests intraepithelium near the basement membrane as a focal matter, then clonal growth of cell keratinocytes changes excessively, replacing the normal epithelium. After some time or several years, invasion of the basement membrane of the epithelial tissue indicates the beginning of invasive cancer. Oral premalignancy is a feature of lesions that can be at risk of turning into uncontrolled cell growth and transforming into cancer followed by chaos in normal tissue function.

The pathological process of premalignancy affects the layered squamous epithelium that protects the oral cavity. The main picture seen before the malignancy is epithelial is histologically describing a combination of disorders in cell maturation and cell proliferation disorders. The degree of epithelial dysplasia and carcinoma are mild dysplasia, intermediate dysplasia, severe dysplasia (carcinoma in situ) and carcinoma. There is clear evidence of immunological effects on the development of malignancy, but whether a tumor develops because the failure of an immune recognition or damage mechanism or other responses is still unclear but it is reported that the immune response can even stimulate oncogenesis. Primary OSCC spreads by local extension through the lymphatic system. Regional spread of the oral mucosa can occur with direct expansion and sometimes with submucosal spread and the result is the extent of the area involved.

Production of type I and other proteinases collagenase, prostaglandin E2, and interleukin 1 can affect the extracellular matrix and motility of epithelial cells can allow invasion. Changes to the basic membrane, such as damage to laminin and collagen, occur with invasion. ${ }^{7,13}$

The American Joint Committee on Cancer (AJCC) has established a cancer staging system with the Tumor-Nodes-Metastasis (TNM) system, which will be a consideration for the prognosis and selection of treatment in cancer cases. $\mathrm{T}$ is the size of the primary tumor, $\mathrm{N}$ indicates involvement of regional lymph glands, and $\mathrm{M}$ indicates the extent of metastasis. The stadium system is a combination of T, N, and M to classify lesions into stages 1 to 4 . The AJCC classification is basically a clinical picture of the disease $(1,14,15)$ in this case, patients initially experience chronic trauma which is later diagnosed with OSCC on the ventrolateral tongue by looking at the clinical condition and FNA examination results categorized as reaching stage III with the TNM system showing T1N1M0 means that a tumor measuring $2 \mathrm{~cm}$ or less was found, $\mathrm{N}$ showed metastasis in a single ipsilateral lymph node, and not distant metastasis. This then made patients and families choose to continue treatment in Penang, Malaysia.

Enforcement of clinical diagnosis in determining the presence of OSCC should still be assisted with investigation. One of the diagnostic tools that can be used in the clinic is Velscope ${ }^{\circledR}$. This tool is a device that produces fluorescent light using an LED source, usually combined with optical filtration, an area suspected of showing loss of fluorescence will appear dark. Fluorescence in the oral cavity, using blue light, is thought to represent tissue structure, metabolic activity, the presence of hemoglobin, dilation of blood vessels, and the possibility of inflammation. Local modification of these factors can change the reflective picture of the network. This product is promoted to help practitioners find mucosal abnormalities, especially potentially malignant abnormalities and evaluate the resection margin. Fluorescence has been shown to provide evidence of lesion margins in patients with known malignant lesions. In this 
case, the patient is also examined using the Velscope ${ }^{\circledR}$ tool. That is one of the autofluorescence devices with LED sources. $(16,17)$ The findings of the patients using this tool which were illuminated in the lesion showed that the bright reddish orange color surface based on the color interpretation did not show any malignancy. This shows that the tool should be routinely calibrated to maintain quality, one possibility is also not to find a dark picture because of the difficulty of affordable lesions using this tool.

The differential diagnosis of OSCC in this case is chronic traumatic ulcer, and erytroleukoplakia. Chronic traumatic ulcer was chosen as one of the differential diagnoses based on history and clinical picture, which according to the patient claimed that the initial appearance of the lesion was often rubbing against the rest of the right maxillary root and sharp lower right tooth surface but after removing the remaining roots, there was no change. Erytroleukoplakia is one of the premalignant lesions that shows white lesions with redness which can be found on the lateral tongue and / or buccal mucosa to the palate, covered with plaques accompanied by redness and pain..$^{11,18,19}$

The first treatment given to this patient was symptomatic by giving Chlorine dioxide mouthwash containing $\mathrm{ClO} 2$. This drug is given before examination of bacterial culture in the lesion. This drug is given to use $5 \mathrm{ml}$ four times a day. This drug is reported to have antibacterial ability by destroying bacterial protein synthesis, deactivating enzymes in bacterial walls, and electrolyte imbalances in cell membranes. ${ }^{20}$ The choice of treatment performed in this case is surgery, a combination of radiotherapy and chemotherapy ${ }^{21}$ Consideration of the treatment is likely caused by the stage of TNM cancer lesions. Postoperative radiotherapy received was 30 times the type of tomoterapi series and 5 times chemotherapy. Thrombotherapy also called "slice therapy " produced electron energy between 4 and $25 \mathrm{MeV}$. The initial management of this tool was initially carried out by NOMOS Corporation. The add-on feature consists of a multileaf collimators set in the form of a narrow fan beam that projects the maximum width of the patient by about $20 \mathrm{~cm}$. The thickness of the fan beam can be 0.8 or $1.6 \mathrm{~cm}$ and each sheet projects a shadow of about $1 \mathrm{~cm}$ in the patient. The sheets will open and close for each slice providing a binary dose delivery, that is, for the beam section, the beam turns on or off. Open beam components are generally referred to as "beamlets" or "beam pencils". Radiation delivery consists of a machine that rotates around the patient when the beam is on and the leaves move in and out quickly, depending on whether the beam is aimed at the target or on normal tissue. After two simultaneous slices have been delivered, the patient is translated into two slice thicknesses and the next two slices are sent until the total volume of treatment is closed, hence it is called "serial tomotherapy". ${ }^{22}$ In addition to physical care, psychological care for patients diagnosed with cancer is needed, especially for families to continue to provide moral support so that patients have the spirit and better life expectancy. This support will improve the patient's ability to survive cancer that is experienced.

\section{Conclusion}

Oral cavity cancer is one of the most feared diseases by the community because it can cause death. Changes in lifestyle and exposure to chemical substances and the role of genetic molecule predisposes to the occurrence of oral cancer. The most common oral cavity cancer is OSCC which is still rare in young men, but early detection can be done with the right tools and methods used to immediately determine patient care. OSCC diagnosis enforcement helps patients to increase life expectancy to survive against cancer, which of course is also not free from family support.

\section{Acknowledgment}

The author would like to thank Prof. Dr. Diah Savitri, drg., M.Sc., Sp.PM as head of the FKG UNAIR HDI Department, to Hening Tuti Hendarti, drg., MS., Sp. PM as Chair of FKG UNAIR's PPDGS IPM Study Program, and Adiastuti, drg., M. Kes., Sp. PM for his guidance in writing and completing this case report.

\section{Conflict of Interest}

The authors report no conflict of interest.

\section{References}

1. Glick M, Feagan CW. Burket's oral medicine. 12th Eedit. Glick M, Feagan Chair W, editors. People's Medical Publishing House. USA: People's Medical Publishing House-USA Shelton, Connecticut; 2015.

2. Ghom AG, Ghom SA. Oral Medicine. In: Ghom AG, Ghom SA, editors. Text book of Oral Medicine. 3rd ed. New Delhi: JaypeeBrothers Medical Publishers; 2014. p. 154-60.

3. Multani R, Shahi P, Yadav A, Yadav S, Setia V. Early Invasive Squamous Cell Carcinoma of the Tongue-A Case Report. Int J Enhanc Res Med Dent Care 2014;1: 1-4.

4. Feller L, Lemmer J. Oral Squamous Cell Carcinoma: Epidemiology, Clinical Presentation and Treatment. J Cancer Ther 2012;3: 263-268. 
5. Badan Penelitian dan Pengembangan Kesehatan. Riset Kesehatan Dasar (RISKESDAS) 2007. Lap Nas 2007. 2008;1: 384.

6. Aliyah SH, To'bungan N, Fachiroh J, Wijayanti N. Usia pasien kaitannya dengan klinikopatologi squamous cell carcinoma (SCC) rongga mulut. Ris Inf Kesehat 2015;5: 124-30.

7. Mehrotra R, Yadav S. Oral squamous cell carcinoma: etiology, pathogenesis and prognostic value of genomic alterations. Indian J Cancer 2006;43: 60-66.

8. Algowaifly M, Alhadlaq RK. Oral squamous cell carcinoma on the lateral border of the tongue: a case report. Dent 2016;6: 10-11.

9. Kayal L, Jayachandran S, Bhaskar HY. Squamous Cell Carcinoma of tongue-a case report and review of literature. Int J Cur Res Rev. 2016;8: 64-67.

10. Brad W. Oral cancer and precancerous lesions. 2002;52: 195-215.

11. Ogbureke KUE. Oral cancer. British Dent J Croatia 2012;181: 129-173.

12. DeLong L, Burkhart NW. General and oral pathology for the dental hygienist. 2nd ed. Philadelphia: Lippincott Williams \& Wilkins; 2013. p. 700.

13. Choi S, Myers JN. Molecular pathogenesis of oral squamous cell carcinoma: Implications for therapy. J Dent Res 2008;87: 14-32.

14. Vasconcelos MG, Mafra RP, Vasconcelos RG, et al. Squamous cell carcinoma of the tongue /: clinical and morphological analysis of 57 cases and correlation with prognosis. J Bras Patol e Med 2014;50: 359-363.

15. Shaeran T, Rahman S, Pohci A, et al. Tackling squamous cell carcinoma of tongue in a 76 Year Old Man: a case report. Bangladesh J Med Sci 2015;14: 413-416.

16. Awan KH, Morgan PR, Warnakulasuriya S. Evaluation of an autofluorescence based imaging system (VELscope ${ }^{\mathrm{TM}}$ ) in the detection of oral potentially malignant disorders and benign keratoses. Oral Oncol 2011;47: 274-277.
17. Balevi B. Assessing the usefulness of three adjunctive diagnostic devices for oral cancer screening: a probabilistic approach. Community Dent Oral Epidemiol 2011;39: 171-176.

18. Bruch MJ, Treiser NS. Clinical oral medicine and pathology. USA: Human Press; 2010. p. 113-28.

19. Eversole LR. Clinical Outline of Oral Pathology /: Diagnosis and Treatment. 4th ed. Malley J, editor. USA: People's Medical Publishing House-USA Shelton Connecticut; 2011.

20. Yeturu SK, Acharya S, Urala AS, et al. Effect of aloe vera, chlorine dioxide and chlorhexidine mouth rinses on plaque and gingivitis: a randomized controlled trial. J Oral Biol Craniofacial Res 2016;6: 55-59.

21. Schmitd LB, Tijoe EC, Assao A, et al. Oral squamous cell carcinoma in young population - risk factors, clinical presentation, and prognosis. In: contemporary issues in head and neck cancer Management. London: Intech; 2015. p. 131-149.

22. Fauzi A, Hardianto A, Wariz R. Hemiglosectomy with selective neck dissection in squamous cell carcinoma at tongue. J Dentomaxillofac Sci 2016;1: 139-144.

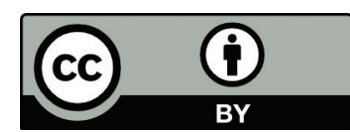

This work is licensed under a Creative Commons Attribution 